\title{
Existence of optimal solutions to Lagrange problems for ordinary control systems involving fractional Laplace operators
}

\section{Rafał Kamocki $^{1}$ (D)}

Received: 24 November 2019 / Accepted: 21 May 2020 / Published online: 31 May 2020

(c) The Author(s) 2020

\begin{abstract}
In this paper, we study optimal control problems containing ordinary control systems, linear with respect to a control variable, described by fractional Dirichlet and Dirichlet-Neumann Laplace operators and a nonlinear integral performance index. The main result is a theorem on the existence of optimal solutions for such problems. In our approach we use a characterization of a weak lower semicontinuity of integral functionals.
\end{abstract}

Keywords Spectral representation of a self-adjoint operator - Dirichlet and Dirichlet-Neumann boundary conditions · Fractional Laplace operator · Existence of optimal solutions $\cdot$ Lower semicontinuity of integral functionals

\section{Introduction}

During the last few decades fractional calculus has been attracted the intersts of many scientists. Recent investigations shown that many phenomena can be accurately described by using differential operators of fractional orders. There exist a lot of types of such operators. In particular, fractional Laplace operators appear in many fields of science; for example in economics [2,15], probability [2,6,7,14], mechanics [5,7], material science [4], fluid mechanics and hydrodynamics [8,11-13,28-30].

They can be defined in many ways (e.g. Fourier transform [16,17], hypersingular integral [16], Riesz potential operator [23], Bochner's definition [27], spectral decomposition $[3,15])$.

Rafał Kamocki

rafal.kamocki@wmii.uni.lodz.pl

1 Faculty of Mathematics and Computer Science, University of Lodz, Banacha 22, 90-238 Lodz, Poland 
Another field of research, in which fractional Laplacians appear, is optimal control theory. In [21], the following two optimal control problems are studied:

$$
\begin{cases} \begin{cases}\left(\left(-\Delta_{k}\right)^{\beta}\right) x(t)=g(t, x(t), u(t)), & t \in(0, \pi) \text { a.e. } \\ u(t) \in M \subset \mathbb{R}^{m}, & t \in(0, \pi),\end{cases} \\ J(x, u)=\int_{0}^{\pi} f_{0}(t, x(t), u(t)) d t \rightarrow \min \end{cases}
$$

where $k=1,2, \beta>\frac{1}{4}, g:(0, \pi) \times \mathbb{R}^{n} \times M \rightarrow \mathbb{R}^{n}$ and $f_{0}:(0, \pi) \times \mathbb{R}^{n} \times M \rightarrow \mathbb{R}$. Here, the control system $\left(E q_{1}\right)$ is described by the one-dimensional Dirichlet Laplace operator $\left(-\Delta_{1}\right)^{\beta}$ of order $\beta$, while $\left(E q_{2}\right)$ involves the Dirichlet-Neumann Laplace operator $\left(-\Delta_{2}\right)^{\beta}$. These operators are defined through the spectral decomposition of the Laplace operator $-\Delta$ in $(0, \pi)$ with zero Dirichlet and Dirichlet-Neumann boundary conditions, respectively (cf. Sect. 2). The main result, obtained in cited paper, are the necessary optimality conditions for the problems $\left(P_{k}\right), k=1,2$ (Pontryagin maximum principle).

In this paper we study the existence of optimal solutions of problems $\left(P_{k}\right), k=1,2$, where $\beta>\frac{1}{2}$ and control systems $\left(E q_{1}\right)$ and $\left(E q_{2}\right)$ are linear with respect to the control variable $u$. Precisely, we consider the following problems:

$$
\begin{cases} \begin{cases}\left(-\Delta_{k}\right)^{\beta} x(t)=f(t, x(t))+B(t) u(t), & t \in(0, \pi) \text { a.e. } \\ u(t) \in M \subset \mathbb{R}^{m}, & t \in(0, \pi)\end{cases} \\ J(x, u)=\int_{0}^{\pi} f_{0}(t, x(t), u(t)) d t \rightarrow \min ,\end{cases}
$$

where $k=1,2, B:(0, \pi) \rightarrow \mathbb{R}^{n \times m}, f:(0, \pi) \times \mathbb{R}^{n} \rightarrow \mathbb{R}^{n}$ and $f_{0}:(0, \pi) \times \mathbb{R}^{n} \times$ $M \rightarrow \mathbb{R}$.

Our study is based on the $L^{1}$ weak lower-semicontinuity of integral functionals [26]. The existence of optimal solutions is also investigated in [9], where an optimal control problem with a fractional Dirichlet Laplacian, defined in $\mathbb{R}^{n}$ is considered. The control system, studied there, has a variational structure and the cost functional depends also on the fractional Laplacian.

The paper is organized as follows. In Sect. 2, we give necessary notions and facts concerning ordinary Dirichlet and Dirichlet-Neumann Laplace operators of fractional order. In Sect. 3, based on a some version of a global implicit function theorem [18], we formulate and prove a theorem on the existence of a unique solution of the control sysytems $\left(E_{k}\right), k=1,2$. In Sect. 4 , we derive the main result of this paper, namely a theorem on the existence of optimal solutions for problems $\left(\mathrm{OCP}_{k}\right), k=1,2$. Section 5 contains an illustrative, theoretical example. We finish with Sect. A containing some basics from the spectral theory of self-adjoint operators in a real Hilbert space. 


\section{Preliminaries}

This part of the paper concerns fractional ordinary Dirichlet and mixed DirichletNeumann Laplace operators. Definitions of these operators are based on the spectral integral representation theorem for a self-adjoint operator in a Hilbert space (cf. [19] and Appendix A).

\section{One-dimensional Dirichlet and Dirichlet-Neumann}

\section{Laplace operators of fractional order}

Let us consider the one-dimensional Laplace operator $-\Delta$ on the interval $(0, \pi)$ given by

$$
-\Delta u=-u^{\prime \prime}
$$

We define the following spaces of functions:

$$
H_{D}:=H_{0}^{1} \cap H^{2} \quad \text { and } \quad H_{D N}:=\left\{z \in H^{2} ; \quad z(0)=z^{\prime}(\pi)=0\right\},
$$

where $H_{0}^{1}=H_{0}^{1}\left((0, \pi), \mathbb{R}^{n}\right)$ and $H^{2}=H^{2}\left((0, \pi), \mathbb{R}^{n}\right)$ are classical Sobolev spaces. We recall that conditions $z(0)=z(\pi)=0$ (hidden in the definition of $H_{D}$ ) and $z(0)=z^{\prime}(\pi)=0$ are called Dirichlet and Dirichlet-Neumann boundary conditions, respectively. Moreover, $H_{D}$ and $H_{D N}$ are dense subspeces of the space $L^{2}=L^{2}\left((0, \pi), \mathbb{R}^{n}\right)$.

The operator $-\Delta: H_{D} \subset L^{2} \rightarrow L^{2}$ given by (1) under Dirichlet boundary conditions is called the Dirichlet Laplace operator and denoted by $-\Delta_{D}$. Similarly, by the Dirichlet-Neumann Laplace operator $-\Delta_{D N}: H_{D N} \subset L^{2} \rightarrow L^{2}$ we mean the operator $-\Delta$ under Dirichlet-Neumann boundary conditions.

In an elementary way one can show that operators $-\Delta_{D}$ and $-\Delta_{D N}$ are self-adjoint. Moreover, their spectrum is given by

$$
\begin{aligned}
\sigma\left(-\Delta_{D}\right) & =\sigma_{p}\left(-\Delta_{D}\right)=\left\{j^{2} ; \quad j=1,2, \ldots\right\}, \\
\sigma\left(-\Delta_{D N}\right) & =\sigma_{p}\left(-\Delta_{D N}\right)=\left\{\left(j-\frac{1}{2}\right)^{2} ; \quad j=1,2, \ldots\right\},
\end{aligned}
$$

respectively and the eigenspaces $\operatorname{Eig}_{j}\left(-\Delta_{D}\right)$ (associated with the eigenvalues $\lambda_{j}=$ $\left.j^{2}\right), \operatorname{Eig}_{j}\left(-\Delta_{D N}\right)$ (associated with the eigenvalues $\lambda_{j}=\left(j-\frac{1}{2}\right)^{2}$ ) are sets

$$
\begin{aligned}
\operatorname{Eig}_{j}\left(-\Delta_{D}\right) & =\left\{c \sin j t ; \quad c \in \mathbb{R}^{n}\right\}, \\
\operatorname{Eig}_{j}\left(-\Delta_{D N}\right) & =\left\{d \sin \left(j-\frac{1}{2}\right) t ; \quad d \in \mathbb{R}^{n}\right\} .
\end{aligned}
$$


It is well known that systems of functions

$$
\begin{aligned}
& c_{i, j}=(0, \ldots, 0, \underbrace{\sqrt{\frac{2}{\pi}} \sin j t}_{i}, 0, \ldots, 0), \quad i=1, \ldots, n, j=1,2, \ldots, \\
& d_{i, j}=(0, \ldots, 0, \underbrace{\sqrt{\frac{2}{\pi}} \sin \left(j-\frac{1}{2}\right)}_{i} t, 0, \ldots, 0), i=1, \ldots, n, j=1,2, \ldots
\end{aligned}
$$

are complete orthonormal systems in $L^{2}$.

Now, let us assume that $\beta>0$. We define the operator

$$
\left(-\Delta_{D}\right)^{\beta}: D\left(\left(-\Delta_{D}\right)^{\beta}\right) \subset L^{2} \rightarrow L^{2}
$$

in the following way (cf. [19, Theorem 2.1])

$$
\left(-\Delta_{D}\right)^{\beta} x(t)=\left(\int_{\sigma\left(-\Delta_{D}\right)} \lambda^{\beta} E(d \lambda) x\right)(t)=\sum_{j=1}^{\infty}\left(j^{2}\right)^{\beta} a_{j} \sqrt{\frac{2}{\pi}} \sin j t
$$

for $x \in D\left(\left(-\Delta_{D}\right)^{\beta}\right)$, where

$$
\begin{aligned}
D\left(\left(-\Delta_{D}\right)^{\beta}\right)=\{x(t)= & \left(\int_{\sigma\left(-\Delta_{D}\right)} 1 E(d \lambda) x\right)(t)=\sum_{j=1}^{\infty} a_{j} \sqrt{\frac{2}{\pi}} \sin j t \in L^{2} ; \\
& \left.\int_{\sigma\left(-\Delta_{D}\right)}\left|\lambda^{\beta}\right|^{2}\|E(d \lambda) x\|_{L^{2}}^{2}=\sum_{j=1}^{\infty}\left(\left(j^{2}\right)^{\beta}\right)^{2}\left|a_{j}\right|^{2}<\infty\right\}
\end{aligned}
$$

(here $E$ is the spectral measure for the operator $-\Delta_{D}$ and $a_{j} \sqrt{\frac{2}{\pi}} \sin j t$ is the projection of $x$ on the $n$-dimensional eigenspace $\left.\operatorname{Eig}_{j}\left(-\Delta_{D}\right)\right)$.

The operator $\left(-\Delta_{D}\right)^{\beta}$ is called the fractional Dirichlet Laplace operator of order $\beta$ and the function $\left(-\Delta_{D}\right)^{\beta} x$ - the fractional Dirichlet Laplacian of order $\beta$ of $x$.

Similarly, we define the fractional Dirichlet-Neumann Laplace operator of order $\beta$

$$
\left(-\Delta_{D N}\right)^{\beta}: D\left(\left(-\Delta_{D N}\right)^{\beta}\right) \subset L^{2} \rightarrow L^{2} .
$$


It is given by

$\left(-\Delta_{D N}\right)^{\beta} x(t)=\left(\int_{\sigma\left(-\Delta_{D N}\right)} \lambda^{\beta} F(d \lambda) x\right)(t)=\sum_{j=1}^{\infty}\left(\left(j-\frac{1}{2}\right)^{2}\right)^{\beta} b_{j} \sqrt{\frac{2}{\pi}} \sin \left(j-\frac{1}{2}\right) t$

for $x \in D\left(\left(-\Delta_{D N}\right)^{\beta}\right)$, where

$$
\begin{aligned}
D\left(\left(-\Delta_{D N}\right)^{\beta}\right)= & \left\{x(t)=\left(\int_{\sigma\left(-\Delta_{D N}\right)} 1 F(d \lambda) x\right)(t)=\sum_{j=1}^{\infty} b_{j} \sqrt{\frac{2}{\pi}} \sin \left(j-\frac{1}{2}\right) t \in L^{2} ;\right. \\
& \left.\int_{\sigma\left(-\Delta_{D N}\right)}\left|\lambda^{\beta}\right|^{2}\|F(d \lambda) x\|_{L^{2}}^{2}=\sum_{j=1}^{\infty}\left(\left(\left(j-\frac{1}{2}\right)^{2}\right)^{\beta}\right)^{2}\left|b_{j}\right|^{2}<\infty\right\}
\end{aligned}
$$

(here $F$ is the spectral measure for the operator $-\Delta_{D N}$ and $b_{j} \sqrt{\frac{2}{\pi}} \sin \left(j-\frac{1}{2}\right) t$ is the projection of $x$ on the $n$-dimensional eigenspace $\operatorname{Eig}_{j}\left(-\Delta_{D N}\right)$ ).

Remark 1 To shorten the notation, in the rest of this paper the fractional Dirichlet (Dirichlet-Neumann) Laplace operator of order $\beta$ is denoted by $\left(-\Delta_{1}\right)^{\beta}\left(\left(-\Delta_{2}\right)^{\beta}\right)$.

Now, we formulate some useful facts concerning mentioned operators and their domains (cf. [19]).

Lemma 1 The spaces $D\left(\left(-\Delta_{k}\right)^{\beta}\right), k=1,2$ are complete with the scalar products

$$
\langle x, y\rangle_{k_{\beta}}=\langle x, y\rangle_{L^{2}}+\left\langle\left(-\Delta_{k}\right)^{\beta} x,\left(-\Delta_{k}\right)^{\beta} y\right\rangle_{L^{2}}, \quad k=1,2 .
$$

The above result follows from the fact that operators $\left.\left(-\Delta_{k}\right)^{\beta}\right), k=1,2$ are selfadjoint, so also closed.

In our paper we shall use a scalar products given by

$$
\langle x, y\rangle_{k_{\sim \beta}}=\left\langle\left(-\Delta_{k}\right)^{\beta} x,\left(-\Delta_{k}\right)^{\beta} y\right\rangle_{L^{2}}, \quad k=1,2,
$$

which generate equivalent norms $\|\cdot\|_{k_{\beta}}$ and $\|\cdot\|_{k \sim \beta}$ in $D\left(\left(-\Delta_{k}\right)^{\beta}\right)$ due to the following Poincaré inequalities:

$$
\begin{aligned}
& \|x\|_{L^{2}}^{2} \leq\|x\|_{1 \sim \beta}^{2}, \quad x \in D\left(\left(-\Delta_{1}\right)^{\beta}\right) \\
& \|x\|_{L^{2}}^{2} \leq 16^{\beta}\|x\|_{2 \sim \beta}^{2}, \quad x \in D\left(\left(-\Delta_{2}\right)^{\beta}\right) .
\end{aligned}
$$


The proof of (3) can be found in [19, formula (11)]. Analogously, we prove inequality (4):

$$
\begin{aligned}
\|x\|_{L^{2}}^{2} & =\sum_{j=1}^{\infty}\left|b_{j}\right|^{2} \leq \sum_{j=1}^{\infty}\left(\left((2 j-1)^{2}\right)^{\beta}\right)^{2}\left|b_{j}\right|^{2} \\
& =16^{\beta} \sum_{j=1}^{\infty}\left(\left(\left(j-\frac{1}{2}\right)^{2}\right)^{\beta}\right)^{2}\left|b_{j}\right|^{2}=16^{\beta}\|x\|_{2 \sim \beta}^{2}, \quad x \in D\left(\left(-\Delta_{2}\right)^{\beta}\right) .
\end{aligned}
$$

Lemma 2 If $\beta>\frac{1}{4}$ then

$$
\begin{aligned}
& \|x\|_{L^{\infty}} \leq \sqrt{\frac{2}{\pi} \zeta(4 \beta)}\|x\|_{1 \sim \beta}, \quad x \in D\left(\left(-\Delta_{1}\right)^{\beta}\right), \\
& \|x\|_{L^{\infty}} \leq 4^{\beta} \sqrt{\frac{2}{\pi} \zeta(4 \beta)}\|x\|_{2 \sim \beta}, \quad x \in D\left(\left(-\Delta_{2}\right)^{\beta}\right),
\end{aligned}
$$

so embeddings

$$
D\left(\left(-\Delta_{k}\right)^{\beta}\right) \subset L^{\infty}, \quad k=1,2
$$

are continuous (here $\zeta$ is the Riemann zeta function given by $\zeta(\gamma)=\sum_{k=1}^{\infty} \frac{1}{k^{\gamma}}$ ).

Proof For the convenience of the reader, we recall the proof of the inequality (6) which can be found in [21] (the proof of (5) for $n=1$ can be found in [19]).

Let $x \in D\left(\left(-\Delta_{2}\right)^{\beta}\right)$. Then

$$
\begin{aligned}
|x(t)|^{2} & =\left|\sum_{j=1}^{\infty} b_{j} \sqrt{\frac{2}{\pi}} \sin \left(j-\frac{1}{2}\right) t\right|^{2}=\sum_{i=1}^{n}\left|\sum_{j=1}^{\infty} b_{j}^{i} \sqrt{\frac{2}{\pi}} \sin \left(j-\frac{1}{2}\right) t\right|^{2} \\
& \leq \frac{2}{\pi} \sum_{i=1}^{n}\left(\sum_{j=1}^{\infty}\left|b_{j}^{i}\right|\right)^{2}=\frac{2}{\pi} \sum_{i=1}^{n}\left(\sum_{j=1}^{\infty} \frac{\left(\left(j-\frac{1}{2}\right)^{2}\right)^{\beta}\left|b_{j}^{i}\right|}{\left(\left(j-\frac{1}{2}\right)^{2}\right)^{\beta}}\right)^{2} \\
& \leq \frac{2}{\pi}\left(\sum_{j=1}^{\infty} \frac{1}{\left(\left(\left(j-\frac{1}{2}\right)^{2}\right)^{\beta}\right)^{2}}\right) \sum_{i=1}^{n}\left(\sum_{j=1}^{\infty}\left(\left(\left(j-\frac{1}{2}\right)^{2}\right)^{\beta}\right)^{2}\left|b_{j}^{i}\right|^{2}\right) \\
& \leq \frac{2}{\pi}\|x\|_{2 \sim \beta}^{2}\left(\sum_{j=1}^{\infty} \frac{1}{\left(\left(\left(j-\frac{1}{2} j\right)^{2}\right)^{\beta}\right)^{2}}=\frac{2}{\pi}\|x\|_{2 \sim \beta}^{2}\left(\sum_{j=1}^{\infty} \frac{1}{\left(\left(\left(\frac{1}{2} j\right)^{2}\right)^{\beta}\right)^{2}}\right)\right. \\
& =\left(4^{\beta}\right)^{2} \frac{2}{\pi}\|x\|_{2 \sim \beta}^{2} \zeta(4 \beta)<\infty, \quad t \in(0, \pi) \text { a.e. }
\end{aligned}
$$

Hence, we obtain inequality (6).

The proof is completed. 
Lemma 3 If $\beta>\frac{1}{2}$ then the operators

$$
\left[\left(-\Delta_{k}\right)^{\beta}\right]^{-1}: L^{2} \ni g \rightarrow x_{g} \in L^{2}, \quad k=1,2
$$

are compact.

Proof The proof of this fact for $k=1$ (in the case of $n=1$ ) is given in [19, proof of Lemma 5.1]. It is analogous for vector valuable functions, so we present only the sketch of it in the case of $k=2$.

Let $F \in L^{2}\left((0, \pi), \mathbb{R}^{n}\right)$ be any bounded (by a constant D) set in $L^{2}\left((0, \pi), \mathbb{R}^{n}\right)$ and consider a function

$$
f(t)=\sum_{j=1}^{\infty} b_{j}^{f} \sqrt{\frac{2}{\pi}} \sin \left(j-\frac{1}{2}\right) t \in F .
$$

In the same way as in $[19$, Section 5.3] we can show that there exists a unique function

$$
x_{f}(t)=\sum_{j=1}^{\infty} c_{j}^{f} \sqrt{\frac{2}{\pi}} \sin \left(j-\frac{1}{2}\right) t
$$

such that

$$
\left(-\Delta_{2}\right)^{\beta} x_{f}(t)=f(t)
$$

Consequently,

$$
\sum_{j=1}^{\infty}\left(\left(j-\frac{1}{2}\right)^{2}\right)^{\beta} c_{j}^{f} \sqrt{\frac{2}{\pi}} \sin \left(j-\frac{1}{2}\right) t=\sum_{j=1}^{\infty} b_{j}^{f} \sqrt{\frac{2}{\pi}} \sin \left(j-\frac{1}{2}\right) t,
$$

so

$$
c_{j}^{f}=\frac{b_{j}^{f}}{\left(\left(j-\frac{1}{2}\right)^{2}\right)^{\beta}} .
$$

Let us consider the set of functions

$$
\mathcal{F}:=\left\{\tilde{x}_{f}: \quad f \in F\right\}
$$

where

$$
\tilde{x}_{f}:(-\infty, \infty) \ni t \rightarrow \begin{cases}x_{f}(t) ; & t \in(0, \pi) \\ 0 ; & \text { otherwise }\end{cases}
$$


Then, for any fixed $h \in(0, \pi)$ we have

$$
\begin{aligned}
\int_{-\infty}^{\infty}\left|\tilde{x}_{f}(t+h)-\tilde{x}_{f}(t)\right|^{2} d t & =\int_{0}^{h}\left|x_{f}(t)\right|^{2} d t+\int_{0}^{\pi-h}\left|x_{f}(t+h)-x_{f}(t)\right|^{2} d t+\int_{\pi-h}^{\pi}\left|x_{f}(t)\right|^{2} d t \\
& =I_{1}+I_{2}+I_{3} .
\end{aligned}
$$

Using the Hölder inequality (for series) we obtain

$$
\begin{aligned}
I_{1} & =\int_{0}^{h} \sum_{i=1}^{n}\left|\sum_{j=1}^{\infty} \frac{\left(b_{j}^{i}\right)^{f}}{\left(\left(j-\frac{1}{2}\right)^{2}\right)^{\beta}} \sqrt{\frac{2}{\pi}} \sin \left(j-\frac{1}{2}\right) t\right|^{2} d t \leq \frac{2}{\pi} \int_{0}^{h} \sum_{i=1}^{n}\left(\sum_{j=1}^{\infty} \frac{\left|\left(b_{j}^{i}\right)^{f}\right|}{\left(\left(j-\frac{1}{2}\right)^{2}\right)^{\beta}}\right)^{2} d t \\
& \leq \frac{2}{\pi} h \sum_{j=1}^{\infty}\left|b_{j}^{f}\right|^{2} \sum_{j=1}^{\infty} \frac{1}{\left(\left(j-\frac{1}{2}\right)^{2}\right)^{2 \beta}} \leq 16^{\beta} \frac{2}{\pi} h\|f\|_{L^{2}}^{2} \zeta(4 \beta) \leq 16^{\beta} \frac{2}{\pi} D \zeta(4 \beta) h .
\end{aligned}
$$

Similarly we estimate the term $I_{3}$. Now, we estimate the term $I_{2}$.

$$
\begin{aligned}
\int_{0}^{\pi-h}\left|x_{f}(t+h)-x_{f}(t)\right|^{2} d t=\int_{0}^{\pi-h}\left|\sum_{j=1}^{\infty} \frac{b_{j}^{f}}{\left(\left(j-\frac{1}{2}\right)^{2}\right)^{\beta}} \sqrt{\frac{2}{\pi}}\left(\sin \left(j-\frac{1}{2}\right)(t+h)-\sin \left(j-\frac{1}{2}\right) t\right)\right|^{2} d t \\
\leq \int_{0}^{\pi-h} \sum_{i=1}^{n}\left(\sum_{j=1}^{\infty} \frac{\left|\left(b_{j}^{i}\right)^{f}\right|}{\left(\left(j-\frac{1}{2}\right)^{2}\right)^{\beta}} \sqrt{\frac{2}{\pi}}\left|2 \sin \frac{\left(j-\frac{1}{2}\right) h}{2} \cos \left(\left(j-\frac{1}{2}\right) t+\frac{\left(j-\frac{1}{2}\right) h}{2}\right)\right|\right)^{2} d t \\
\leq \frac{8}{\pi} \int_{0}^{\pi-h} \sum_{i=1}^{n}\left(\sum_{j=1}^{\infty} \frac{\left|\left(b_{j}^{i}\right)^{f}\right|}{\left(\left(j-\frac{1}{2}\right)^{2}\right)^{\beta}}\left|\sin \frac{\left(j-\frac{1}{2}\right) h}{2}\right|\right)^{2} d t \\
\leq \frac{8}{\pi}(\pi-h) \sum_{j=1}^{\infty}\left|b_{j}^{f}\right|^{2} \sum_{j=1}^{\infty} \frac{\sin ^{2} \frac{\left(j-\frac{1}{2}\right) h}{2}}{\left(\left(j-\frac{1}{2}\right)^{2}\right)^{2 \beta}} \leq 8 D \sum_{j=1}^{\infty} \frac{\left(j-\frac{1}{2}\right) h}{2\left(\left(j-\frac{1}{2}\right)^{2}\right)^{2 \beta}} \\
\quad=4 D h \sum_{j=1}^{\infty} \frac{1}{\left(j-\frac{1}{2}\right)^{4 \beta-1}}=2 D 16^{\beta} \zeta(4 \beta-1) h .
\end{aligned}
$$

Analogously, we estimate terms $I_{1}, I_{2}, I_{3}$ for any fixed $h \in(-\pi, 0)$.

Finally,

$$
\int_{-\infty}^{\infty}\left|\tilde{x}_{f}(t+h)-\tilde{x}_{f}(t)\right|^{2} d t \leq \text { const }|h|, \quad|h| \leq \pi .
$$

Consequently,

$$
\lim _{|h| \rightarrow 0}\left\|\tau_{h} \tilde{x}-\tilde{x}\right\|_{L^{2}\left(\mathbb{R}, \mathbb{R}^{n}\right)}=0 \text { uniformly on } \mathcal{F}
$$


where $\tau_{h} \tilde{x}(t)=\tilde{x}(t+h)$, so the set

$$
\left.\mathcal{F}\right|_{(0, \pi)}=\left\{x_{f}: \quad f \in F\right\}
$$

is relatively compact in $L^{2}\left((0, \pi), \mathbb{R}^{n}\right)$.

The proof is completed.

Remark 2 The relatively compactness of $\mathcal{F}$ follows from the following KolmogorovFréchet-Riesz theorem (cf. [10, Theorem 4.26]):

Theorem 1 (Kolmogorov-Fréchet-Riesz) Let $\mathcal{F}$ be a bounded set in $L^{p}\left(\mathbb{R}^{N}\right)$ with $1 \leq p<\infty$. Assume that

$$
\lim _{|h| \rightarrow 0}\left\|\tau_{h} f-f\right\|_{L^{p}}=0 \quad \text { uniformly in } f \in \mathcal{F},
$$

i.e.

$$
\forall_{\varepsilon>0} \exists_{\delta>0} \text { such that }\left\|\tau_{h} f-f\right\|_{L^{p}}<\varepsilon, \quad \forall_{f \in \mathcal{F}}, \quad \forall_{h \in \mathbb{R}^{N}} \text { with }|h|<\delta .
$$

Then the closure of $\mathcal{F}_{\mid \Omega}$ in $L^{p}\left(\mathbb{R}^{N}\right)$ is compact for any measurable set $\Omega \subset \mathbb{R}^{N}$ with finite measure.

(Here $\mathcal{F}_{\mid \Omega}$ denotes the restrictions to of the functions in $\mathcal{F}$ ).

Using the above lemma and analogous arguments as in the proof of [19, Lemma 5.2] we obtain

Corollary 1 Let $k=1,2$ and $\beta>\frac{1}{2}$. If $x_{n} \rightarrow x_{0}$ weakly in $D\left(\left(-\Delta_{k}\right)^{\beta}\right)$ then $x_{n} \rightarrow x_{0}$ strongly in $L^{2}$ and $\left(-\Delta_{k}\right)^{\beta} x_{n} \rightarrow\left(-\Delta_{k}\right)^{\beta} x_{0}$ weakly in $L^{2}$.

\section{Existence and uniqueness of a solution to the control systems $\left(E_{1}\right)$ and $\left(E_{2}\right)$}

The main result of this section is a theorem on the existence of a unique solution to the control systems $\left(\mathrm{E}_{k}\right), k=1,2$. In the proof of this fact we use the following result.

Theorem 2 (Corollary 3.3, [18]) Let $X$ be a real Banach space, $Y$ a non-empty set, and $H$ a real Hilbert space. If $F: X \times Y \rightarrow H$ is continuously differentiable with respect to $x \in X$ and

- for any $y \in Y$ the functional

$$
\phi_{y}: X \ni x \rightarrow \frac{1}{2}\|F(x, y)\|^{2} \in \mathbb{R}
$$

satisfies the Palais-Smale (PS) condition ${ }^{1}$,

\footnotetext{
${ }^{1}$ Let $X$ be a real Banach space and $I: X \rightarrow \mathbb{R}$ denotes a functional of class $C^{1}$. We say that $I$ satisfies the Palais-Smale (PS) condition if any sequence $\left(x_{l}\right)_{l \in \mathbb{N}}$ such that
} 
$-F_{x}^{\prime}(x, y): X \rightarrow Y$ is bijective for any $(x, y) \in X \times Y$ such that $F(x, y)=0$ and

$$
F(x, y) \notin\left(\operatorname{Im}_{x}^{\prime}(x, y)\right)^{\perp}
$$

for any $(x, y) \in X \times Y$ such that $F(x, y) \neq 0$

then, for any $y \in Y$, there exists a unique $x_{y} \in X$ such that $F\left(x_{y}, y\right)=0$.

In the rest of this paper we assume that $\beta>\frac{1}{2}$. Let us define the following set of controls:

$$
\mathcal{U}_{M}:=\left\{u \in L^{2}\left((0, \pi), \mathbb{R}^{m}\right) ; \quad u(t) \in M, \quad t \in(0, \pi)\right\}
$$

We have

Theorem 3 Let us fix $k=1$, 2. If

(A1) $f$ is measurable in $t \in(0, \pi)$, continuously differentiable in $x \in \mathbb{R}^{n}$ and

$$
\begin{aligned}
& |f(t, x)| \leq a(t)|x|+b(t), \quad t \in(0, \pi) \text { a.e., } x \in \mathbb{R}^{n}, \\
& \left|f_{x}(t, x)\right| \leq a(t) \delta(|x|), \quad t \in(0, \pi) \text { a.e., } x \in \mathbb{R}^{n},
\end{aligned}
$$

where $\delta \in C\left(\mathbb{R}_{0}^{+}, \mathbb{R}_{0}^{+}\right)$and $a, b \in L^{2}\left((0, \pi), \mathbb{R}^{+}\right)$is such that

$$
\begin{aligned}
& \sqrt{\frac{2}{\pi} \zeta(4 \beta)}\|a\|_{L^{2}}<1 \quad \text { if } \quad k=1 \\
& \sqrt{\frac{2}{\pi} \zeta(4 \beta)}\|a\|_{L^{2}}<\frac{1}{4^{\beta}} \quad \text { if } \quad k=2,
\end{aligned}
$$

(A2) $B \in L^{\infty}\left((0, \pi), \mathbb{R}^{n \times m}\right)$

(A3) for any pair $(x, u) \in D\left(\left(-\Delta_{k}\right)^{\beta}\right) \times \mathcal{U}_{M}$ one of the following three conditions are satisfied

(a)

$$
\begin{aligned}
& \|\Lambda\|_{L^{1}} \leq \frac{\pi}{2 \zeta(2 \beta)} \quad \text { if } \quad k=1, \\
& \|\Lambda\|_{L^{1}} \leq \frac{\pi}{2 \zeta(2 \beta) 4^{\beta}} \quad \text { if } \quad k=2,
\end{aligned}
$$

(b) $\Lambda(t) \leq 0, \quad t \in(0, \pi)$ a.e.,

(c) $\Lambda \in L^{\infty}\left((0, \pi), \mathbb{R}^{n \times n}\right)$ and $\|\Lambda\|_{L^{\infty}}<1$,

where $\Lambda(\cdot)=f_{x}(\cdot, x(\cdot))$

- $\left|I\left(x_{l}\right)\right| \leq M$ for all $l \in \mathbb{N}$ and some $M>0$,

- $I^{\prime}\left(x_{l}\right) \rightarrow 0$,

admits a convergent subsequence $\left(I^{\prime}\left(x_{l}\right)\right.$ denotes the Fréchet differential of $I$ at $\left.x_{l}\right)$. 
then for any fixed control $u \in \mathcal{U}_{M}$ there exists a unique solution $x_{u} \in D\left(\left(-\Delta_{k}\right)^{\beta}\right)$ of the control system $\left(E_{k}\right)$.

Proof Let us fix $k=1,2$ and define the operator

$$
F_{k}: D\left(\left(-\Delta_{k}\right)^{\beta}\right) \times \mathcal{U}_{M} \ni(x, u) \rightarrow\left(-\Delta_{k}\right)^{\beta} x(t)-f(t, x(t))-B(t) u(t) \in L^{2} .
$$

It is sufficient to show that $F_{k}$ satisfies all assumptions of Theorem 2.

- Using assumptions (A1), (A2) and analogous arguments as in [19, Proposition 5.1], we check that the mapping $F_{k}$ is continuously differentiable with respect to $x \in D\left(\left(-\Delta_{k}\right)^{\beta}\right)$ and the differential $\left(F_{k}\right)_{x}: D\left(\left(-\Delta_{k}\right)^{\beta}\right) \rightarrow L^{2}$ of $F_{k}$ at the point $(x, u)$ is given by

$$
\left(F_{k}\right)_{x}(x, u) h=\left(-\Delta_{k}\right)^{\beta} h(t)-f_{x}(t, x(t)) h(t)
$$

for any fixed $u \in \mathcal{U}_{M}{ }^{2}$

- (c) Now, we show that for any $u \in \mathcal{U}_{M}$ the functional

$$
\phi_{u}^{k}: D\left(\left(-\Delta_{k}\right)^{\beta}\right) \ni x \rightarrow \frac{1}{2}\left\|F_{k}(x, u)\right\|^{2} \in \mathbb{R}
$$

satisfies the Palais-Smale condition.

First, let us observe that the growth condition (9) and conditions (11), (12) guarantee coercivity of $\phi_{u}^{k}$ for any $u \in \mathcal{U}_{M}$ (it is sufficient to use the same arguments as in the proof of [19, Lemma 5.3]). Moreover, it is continuously differentiable with respect to $x$ and its differential $\left(\phi_{u}^{k}\right)^{\prime}: D\left(\left(-\Delta_{k}\right)^{\beta}\right) \rightarrow \mathbb{R}$ is given by

$$
\left(\phi_{u}^{k}\right)^{\prime}(x) h=\int_{0}^{\pi}\left\langle\left(-\Delta_{k}\right)^{\beta} x(t)-f(t, x(t))-B(t) u(t),\left(-\Delta_{k}\right)^{\beta} h(t)-f_{x}(t, x(t)) h(t)\right\rangle d t
$$

for any $h \in D\left(\left(-\Delta_{k}\right)^{\beta}\right)$. Let $x_{0} \in D\left(\left(-\Delta_{k}\right)^{\beta}\right)$ and $\left(x_{l}\right)_{l \in \mathbb{N}} \subset D\left(\left(-\Delta_{k}\right)^{\beta}\right)$. Then

$$
\left(\phi_{u}^{k}\right)^{\prime}\left(x_{l}\right)-\left(\phi_{u}^{k}\right)^{\prime}\left(x_{0}\right)\left(x_{l}-x_{0}\right)=\left\|x_{l}-x_{0}\right\|_{\sim \frac{\beta}{2}}^{2}+\sum_{i=1}^{5} \psi_{i}^{k}\left(x_{l}\right),
$$

\footnotetext{
${ }^{2}$ In order to prove a differentiability property of the mapping $F_{2}$ (then $\left(-\Delta_{2}\right)^{\beta}=\left(-\Delta_{D N}\right)^{\beta}$ denotes the Dirichlet-Neumann Laplace operator of order $\beta$ ) we use the estimation (6) instead of (5).
} 
where

$$
\begin{aligned}
\psi_{1}^{k}\left(x_{l}\right)= & \int_{0}^{\pi}\left\langle\left(-\Delta_{k}\right)^{\beta} x_{l}(t), f_{x}\left(t, x_{l}(t)\right)\left(x_{0}(t)-x_{l}(t)\right)\right\rangle d t, \\
\psi_{2}^{k}\left(x_{l}\right)= & \int_{0}^{\pi}\left\langle\left(-\Delta_{k}\right)^{\beta} x_{0}(t), f_{x}\left(t, x_{0}(t)\right)\left(x_{l}(t)-x_{0}(t)\right)\right\rangle d t, \\
\psi_{3}^{k}\left(x_{l}\right)= & \int_{0}^{\pi}\left\langle f\left(t, x_{l}(t)\right), f_{x}\left(t, x_{l}(t)\right)\left(x_{l}(t)-x_{0}(t)\right)\right\rangle d t, \\
\psi_{4}^{k}\left(x_{l}\right)= & \int_{0}^{\pi}\left\langle f\left(t, x_{0}(t)\right), f_{x}\left(t, x_{0}(t)\right)\left(x_{0}(t)-x_{l}(t)\right)\right\rangle d t, \\
\psi_{5}^{k}\left(x_{l}\right)= & \int_{0}^{\pi}\left\langle f\left(t, x_{0}(t)\right)-f\left(t, x_{l}(t)\right),\left(-\Delta_{k}\right)^{\beta} x_{l}(t)-\left(-\Delta_{k}\right)^{\beta} x_{0}(t)\right\rangle d t .
\end{aligned}
$$

Using analogous arguments as in the proof of [19, Proposition 5.3] (including coercivity of $\phi_{u}^{k}$, Corollary 1 and the Lebesque dominated convergence theorem) we conclude that there exists a subsequence $\left(x_{l_{j}}\right)_{j \in \mathbb{N}}$ such that

$$
\psi_{i}^{k}\left(x_{l_{j}}\right) \underset{j \rightarrow \infty}{\longrightarrow} 0, \quad i=1, \ldots, 5, \quad k=1,2
$$

This means that $x_{l_{j}} \longrightarrow x_{0}$ in $D\left(\left(-\Delta_{k}\right)^{\beta}\right)$, so for any $u \in \mathcal{U}_{M}$ the functional $\phi_{u}^{k}$ satisfies the Palais-Smale condition.

- Analogously as in [19, Proposition 5.2] (using the assumption (A3)) we show that for any pair $(x, u) \in D\left(\left(-\Delta_{k}\right)^{\beta}\right) \times \mathcal{U}_{M}$ the differential $\left(F_{k}\right)_{x}$ is bijective ${ }^{3}$.

The proof is completed.

We also have the following two results

Proposition 1 If $M$ is a bounded set and assumptions (A1), (A2), (A3) of Theorem 3 are satisfied then there exists constants $C_{1}, C_{2}>0$ (inpedendent on $u$ ) such that for any control $u \in \mathcal{U}_{M}$

$$
\left\|x_{u}\right\|_{k \sim \beta} \leq C_{k}, \quad k=1,2 .
$$

Proof Let us fix $k=1,2$ and any control $u \in \mathcal{U}_{M}$. Let $C$ be a constant such that $|u(t)| \leq C$ for a.e. $t \in(0, \pi)$. Assume that $x_{u} \in D\left(\left(-\Delta_{k}\right)^{\beta}\right)$ is a solution of the

\footnotetext{
3 The proof of the fact that the mapping $\left(F_{2}\right)_{x}\left(x_{*}, u_{*}\right)$ is bijective is analogous to the proof of bijectivity of $\left(F_{1}\right)_{x}\left(x_{*}, u_{*}\right)$ (cf. [19, Proposition 5.2] - the condition (13) is replaced with (14)). 
control system $\left(\mathrm{E}_{k}\right)$, corresponding to $u$. Then, using (9), we obtain

$$
\begin{aligned}
\left\|x_{u}\right\|_{k \sim \beta} & =\left\|\left(-\Delta_{k}\right)^{\beta} x_{u}\right\|_{L^{2}}=\left(\int_{0}^{\pi}\left|f\left(t, x_{u}(t)\right)+B(t) u(t)\right|^{2} d t\right)^{\frac{1}{2}} \\
& \leq\left(\int_{0}^{\pi}|a(t)|^{2}\left|x_{u}(t)\right|^{2} d t\right)^{\frac{1}{2}}+\left(\int_{0}^{\pi}|B(t)|^{2}|u(t)|^{2} d t\right)^{\frac{1}{2}}+\|b\|_{L^{2}} \\
& \leq\|a\|_{L^{2}}\left\|x_{u}\right\|_{L^{\infty}}+C \sqrt{\pi}\|B\|_{L^{\infty}}+\|b\|_{L^{2}} .
\end{aligned}
$$

Thus and from Lemma 2 we have

$$
\left\|x_{u}\right\|_{k \sim \beta} \leq\left\{\begin{array}{l}
\sqrt{\frac{2}{\pi} \zeta(4 \beta)}\|a\|_{L^{2}}\left\|x_{u}\right\|_{k_{\sim \beta}}+C \sqrt{\pi}\|B\|_{L^{\infty}}+\|b\|_{L^{2}} \quad \text { if } \quad k=1 \\
4^{\beta} \sqrt{\frac{2}{\pi} \zeta(4 \beta)}\|a\|_{L^{2}}\left\|x_{u}\right\|_{k_{\sim \beta}}+C \sqrt{\pi}\|B\|_{L^{\infty}}+\|b\|_{L^{2}} \quad \text { if } \quad k=2 .
\end{array}\right.
$$

This means that

$$
\left\|x_{u}\right\|_{k \sim \beta} \leq C_{k}
$$

where

$$
C_{k}= \begin{cases}\frac{\sqrt{\pi} C\|B\|_{L^{\infty}+\|b\|_{L^{2}}}}{1-\sqrt{\frac{2}{\pi} \zeta(4 \beta)\|a\|_{L^{2}}}} & \text { if } \quad k=1 \\ \frac{\sqrt{\pi} C\|B\|_{L^{\infty}+\|b\|_{L^{2}}}}{1-4 \beta \sqrt{\frac{2}{\pi} \zeta(4 \beta)\|a\|_{L^{2}}}} & \text { if }\end{cases}
$$

The proof is completed.

Proposition 2 Let us fix $k=1,2$. Assume that all assumptions of Theorem 3 are satisfied and the set $M$ is convex and compact. If $\left(u_{l}\right)_{l \in \mathbb{N}} \subset \mathcal{U}_{M}$ is a sequence of controls and $\left(x_{l}\right)_{l \in \mathbb{N}} \subset D\left(\left(-\Delta_{k}\right)^{\beta}\right)$ is a sequence of corresponding solutions of the control system $\left(E_{k}\right)$ then there exist a control $u_{0} \in \mathcal{U}_{M}$, a function $x_{0} \in D\left(\left(-\Delta_{k}\right)^{\beta}\right)$ and a subsequence $\left(l_{i}\right)_{i \in \mathbb{N}}$ such that the pair $\left(x_{0}, u_{0}\right)$ satisfies $\left(E_{k}\right)$ and

(Z1) $x_{l_{i}} \underset{i \rightarrow \infty}{\longrightarrow} x_{0}$ strongly in $L^{2}$,

(Z2) $\left(-\Delta_{k}\right)^{\beta} x_{l_{i}} \rightarrow\left(-\Delta_{k}\right)^{\beta} x_{0}$ weakly in $L^{2}$,

(Z3) $u_{l_{i}} \underset{i \rightarrow \infty}{\rightarrow} u_{0}$ weakly in $L^{2}\left((0, \pi), \mathbb{R}^{m}\right)$.

Proof Let us fix $k=1,2$ and consider a sequence of controls $\left(u_{l}\right)_{l \in \mathbb{N}} \in \mathcal{U}_{M}$ and a sequence of corresponding solutions $\left(x_{l}\right)_{l \in \mathbb{N}} \subset D\left(\left(-\Delta_{k}\right)^{\beta}\right)$ of the system $\left(E_{k}\right)$. Using the standard arguments we check that compactness and convexity of the set $M$ imply a convexity, boundedness and closure of the set $\mathcal{U}_{M}$ in $L^{2}\left((0, \pi), \mathbb{R}^{m}\right)$. This means 
that $\mathcal{U}_{M}$ is sequentially weakly compact, while $L^{2}\left((0, \pi), \mathbb{R}^{m}\right)$ is a reflexive space. Consequently, there exist a subsequence $\left(u_{l_{i}}\right)_{i \in \mathbb{N}}$ and $u_{0} \in \mathcal{U}_{M}$ such that

$$
u_{l_{i}} \rightarrow u_{0} \text { weakly in } L^{2}\left((0, \pi), \mathbb{R}^{m}\right)
$$

so the condition (Z3) of this proposition is satisfied.

From Proposition 1 it follows that the sequence of norms $\left\|x_{l}\right\|_{k_{\sim \beta}}$ is bounded, so, there exist a subsequence $\left(x_{l_{i}}\right)_{i \in \mathbb{N}}$ and a function $x_{0} \in D\left(\left(-\Delta_{k}\right)^{\beta}\right)$ such that

$$
x_{l_{i}} \underset{i \rightarrow \infty}{\rightarrow} x_{0} \text { weakly in } D\left(\left(-\Delta_{k}\right)^{\beta}\right) .
$$

Consequently, Corollary 1 implies convergences (Z1) and (Z2).

Now, we show that the $x_{0}$ is a solution of $\left(E_{k}\right)$, corresponding to $u_{0}$. Indeed, first we note that since the matrix $B$ is essentially bounded on $(0, \pi)$, therefore

$$
B u_{l_{i}} \rightarrow B u_{0} \text { weakly in } L^{2}
$$

Moreover, using condition (9) and Lemma 2 we have

$$
\begin{aligned}
\left|f\left(t, x_{l_{i}}(t)\right)-f\left(t, x_{0}(t)\right)\right|^{2} & \leq 2\left(\left|f\left(t, x_{l_{i}}(t)\right)\right|^{2}+\left|f\left(t, x_{0}(t)\right)\right|^{2}\right) \\
& \leq 2 a^{2}(t)\left(\left\|x_{l_{i}}\right\|_{L^{\infty}}^{2}+\left|x_{0}(t)\right|^{2}\right) \\
& \leq\left\{\begin{array}{l}
2 a^{2}(t)\left(\frac{2}{\pi} \zeta(4 \beta)\left\|x_{l_{i}}\right\|_{k_{\sim \beta}}^{2}+\left|x_{0}(t)\right|^{2}\right) \quad \text { if } k=1 \\
2 a^{2}(t)\left(16^{\beta} \frac{2}{\pi} \zeta(4 \beta)\left\|x_{l_{i}}\right\|_{k_{\sim \beta}}^{2}+\left|x_{0}(t)\right|^{2}\right) \quad \text { if } k=2
\end{array}\right. \\
& \leq \begin{cases}2 a^{2}(t)\left(\frac{2}{\pi} \zeta(4 \beta) C_{k}^{2}+\left|x_{0}(t)\right|^{2}\right) & \text { if } k=1 \\
2 a^{2}(t)\left(16^{\beta} \frac{2}{\pi} \zeta(4 \beta) C_{k}^{2}+\left|x_{0}(t)\right|^{2}\right) & \text { if } k=2,\end{cases}
\end{aligned}
$$

where $C_{k}, k=1,2$ are constants from Proposition 1. Consequently, from the Lebesque dominated convergence theorem it follows that

$$
f\left(\cdot, x_{l_{i}}(\cdot)\right) \underset{i \rightarrow \infty}{\longrightarrow} f\left(\cdot, x_{0}(\cdot)\right) \text { strongly in } L^{2}
$$

Then, of course

$$
f\left(\cdot, x_{l_{i}}(\cdot)\right) \underset{i \rightarrow \infty}{\rightarrow} f\left(\cdot, x_{0}(\cdot)\right) \text { weakly in } L^{2} .
$$

Thus, using (Z2) we get

$$
\left(-\Delta_{k}\right)^{\beta} x_{l_{i}}(\cdot)-f\left(\cdot, x_{l_{i}}(\cdot)\right)-B(\cdot) u_{l_{i}}(\cdot) \underset{k \rightarrow \infty}{\rightarrow}\left(-\Delta_{k}\right)^{\beta} x_{0}(\cdot)-f\left(\cdot, x_{0}(\cdot)\right)-B(\cdot) u_{0}(\cdot)
$$


weakly in $L^{2}\left((0, \pi), \mathbb{R}^{n}\right)$. On the other hand, $\left(x_{l_{i}}\right)$ is a solution of $\left(E_{k}\right)$, corresponding to $\left(u_{l_{i}}\right)$, so we have

$$
\left(-\Delta_{k}\right)^{\beta} x_{l_{i}}(t)-f\left(t, x_{l_{i}}(t)\right)-B(t) u_{l_{i}}(t)=0, \quad t \in(0, \pi) \text { a.e. }
$$

This means that

$$
\left(-\Delta_{k}\right)^{\beta} x_{0}(t)-f\left(t, x_{0}(t)\right)-B(t) u_{0}(t)=0, \quad t \in(0, \pi) \text { a.e. }
$$

The proof is completed.

\section{Existence of optimal solutions}

In this section we shall prove the main result of this paper, namely a theorem on the existence of optimal solutions of the problems $\left(\mathrm{OCP}_{k}\right), k=1,2$.

Let us fix $k=1,2$. We shall say, that a pair $\left(x_{*}, u_{*}\right) \in D\left(\left(-\Delta_{k}\right)^{\beta}\right) \times \mathcal{U}_{M}$ is a globally optimal solution of the problem $\left(\mathrm{OCP}_{k}\right)$, if $x_{*}$ is the solution of the control system $\left(E_{k}\right)$, corresponding to the control $u_{*}$ and

$$
J\left(x_{*}, u_{*}\right) \leq J(x, u)
$$

for every pair $(x, u) \in D\left(\left(-\Delta_{k}\right)^{\beta}\right) \times \mathcal{U}_{M}$ satisfying $\left(E_{k}\right)$.

We have

Theorem 4 Let us fix $k=1,2$ and assume that

1. $M$ is convex and compact,

2. hypothesis (A1), (A2) and (A3) of Theorem 3 are satisfied,

3. $f_{0}(\cdot, x, u)$ is measurable on $(0, \pi)$ for all $x \in \mathbb{R}^{n}$ and $u \in M$,

4. $f_{0}(t, \cdot, \cdot)$ is continuous on $\mathbb{R}^{n} \times M$ for a.e. $t \in(0, \pi)$,

5. $f_{0}(t, x, \cdot)$ is convex on $M$ for a.e. $t \in(0, \pi)$ and all $x \in \mathbb{R}^{n}$,

6. there exist a summable function $\psi:(0, \pi) \rightarrow \mathbb{R}_{0}^{+}$and a constant $c \geq 0$ such that

$$
f_{0}(t, x, u) \geq-\psi(t)-c|x|
$$

for a.e. $t \in(0, \pi)$ and all $x \in \mathbb{R}^{n}, u \in M$.

Then the problem $\left(O C P_{k}\right)$ possesses an optimal solution $\left(x_{0}, u_{0}\right) \in D\left(\left(-\Delta_{k}\right)^{\beta}\right) \times \mathcal{U}_{M}$.

Proof Let us fix $k=1,2$ and denote

$$
\mu=\inf \left\{J\left(x_{u}, u\right), \quad u \in \mathcal{U}_{M}\right\}
$$


It is clear that $\mu \leq J\left(x_{u}, u\right)$ for any pair $\left(x_{u}, u\right) \in D\left(\left(-\Delta_{k}\right)^{\beta}\right) \times \mathcal{U}_{M}$. The condition (16), the Hölder inequality, Poincaré inequalities (3), (4) and Proposition 1 imply

$$
\begin{aligned}
J\left(x_{u}, u\right) & =\int_{0}^{\pi} f_{0}\left(t, x_{u}(t), u(t)\right) d t \geq-\int_{0}^{\pi} \psi(t) d t-c \int_{0}^{\pi}\left|x_{u}(t)\right| d t \\
& \geq-\int_{0}^{\pi} \psi(t) d t-c \sqrt{\pi}\left\|x_{u}\right\|_{L^{2}} \\
& \geq-\int_{0}^{\pi} \psi(t) d t- \begin{cases}c \sqrt{\pi}\left\|x_{u}\right\|_{k \sim \beta} & \text { if } k=1 \\
c \sqrt{\pi} 4^{\beta}\left\|x_{u}\right\|_{k \sim \beta} & \text { if } k=2\end{cases} \\
& \geq-\int_{0}^{\pi} \psi(t) d t-\left\{\begin{array}{ll}
c \sqrt{\pi} C_{k} & \text { if } k=1 \\
c \sqrt{\pi} 4^{\beta} C_{k} & \text { if } k=2
\end{array} \quad-\infty,\right.
\end{aligned}
$$

where $C_{1}, C_{2}$ are constants from Proposition 1. This means that $-\infty<\mu \leq+\infty$. If $\mu=+\infty$ then the existence of optimal solutions is obvious.

So, let us assume that $-\infty<\mu<+\infty$ and $\left(x_{l}, u_{l}\right)_{l \in \mathbb{N}} \in D\left(\left(-\Delta_{k}\right)^{\beta}\right) \times \mathcal{U}_{M}$ be a minimizing sequence of the functional $J$. This means that

$$
\lim _{l \rightarrow \infty} J\left(x_{l}, u_{l}\right)=\mu
$$

From Proposition 2 it follows that there exist a pair $\left(x_{0}, u_{0}\right) \in D\left(\left(-\Delta_{k}\right)^{\beta}\right) \times \mathcal{U}_{M}$ and a subsequence $\left(l_{i}\right)_{i \in \mathbb{N}}$ such that the pair $\left(x_{0}, u_{0}\right)$ satisfies $\left(E_{k}\right)$ and

$$
\begin{aligned}
& u_{l_{i}} \rightarrow u_{0} \text { weakly in } L^{2}\left((0, \pi), \mathbb{R}^{m}\right), \\
& x_{l_{i}} \underset{i \rightarrow \infty}{\rightarrow} x_{0} \text { strongly in } L^{2} .
\end{aligned}
$$

Thus, we obtain respective convergences in $L^{1}\left((0, \pi), \mathbb{R}^{m}\right)$ and $L^{1}\left((0, \pi), \mathbb{R}^{n}\right)$, respectively.

Now, let us consider a function $\hat{f}_{0}:(0, \pi) \times \mathbb{R}^{n} \times M \rightarrow \mathbb{R}$ given by

$$
\hat{f_{0}}(t, x, u):= \begin{cases}f_{0}(t, x, u) ; & t \in T \\ -\psi(t)-c|x| ; & t \notin T\end{cases}
$$

where $T \subset(0, \pi)$ is a set of the full measure consist of points, for which conditions $4,5,6$ are satisfied. Then the function $\hat{f}_{0}$ satisfies mentioned conditions for all $t \in$ $(0, \pi)$. From [22, Proposition 3.2] and [24, section IV $\S 3$ Theorem 6] it follows that $\hat{f}_{0}$ is $\mathcal{L}((0, \pi)) \times \mathcal{B}\left(\mathbb{R}^{n} \times M\right)$ measurable. Moreover, it can be extended to the function 
$\tilde{f_{0}}:(0, \pi) \times \mathbb{R}^{n} \times \mathbb{R}^{m} \rightarrow \mathbb{R}$ given by

$$
\tilde{f}_{0}(t, x, u):=\left\{\begin{array}{l}
\hat{f}_{0}(t, x, u) ; \quad(t, x, y) \in(0, \pi) \times \mathbb{R}^{n} \times M \\
+\infty ; \quad(t, x, y) \notin(0, \pi) \times \mathbb{R}^{n} \times M
\end{array}\right.
$$

[20, Lemma 16] guarantees $\mathcal{L}((0, \pi)) \times \mathcal{B}\left(\mathbb{R}^{n} \times \mathbb{R}^{m}\right)$ - measurability of $\tilde{f}_{0}$. This function is also lower semicontinuous with respect to $(x, u) \in \mathbb{R}^{n} \times \mathbb{R}^{m}$ for any fixed $t \in(0, \pi)$, convex with respect to $u \in \mathbb{R}^{m}$ for any fixed $(t, x) \in(0, \pi) \times \mathbb{R}^{n}$ and satisfies inequality (16) for all points $(t, x, u) \in(0, \pi) \times \mathbb{R}^{n} \times \mathbb{R}^{m}$. Consequently, using a theorem on the $L^{1}$-weak lower semicontinuity of integral functionals (cf. [26]) we assert that

$$
\tilde{J}\left(x_{0}, u_{0}\right) \leq \liminf _{i \rightarrow \infty} \tilde{J}\left(x_{l_{i}}, u_{l_{i}}\right)
$$

where

$$
\tilde{J}(x, u)=\int_{0}^{\pi} \tilde{f}_{0}(t, x(t), u(t)) d t .
$$

Thus, since

$$
J\left(x_{0}, u_{0}\right)=\hat{J}\left(x_{0}, u_{0}\right)=\tilde{J}\left(x_{0}, u_{0}\right) \quad \text { and } \quad J\left(x_{l_{i}}, u_{l_{i}}\right)=\hat{J}\left(x_{l_{i}}, u_{l_{i}}\right)=\tilde{J}\left(x_{l_{i}}, u_{l_{i}}\right),
$$

where

$$
\hat{J}(x, u)=\int_{a}^{b} \hat{f}_{0}(t, x(t), u(t)) d t,
$$

therefore

$$
J\left(x_{0}, u_{0}\right) \leq \liminf _{i \rightarrow \infty} J\left(x_{l_{i}}, u_{l_{i}}\right)
$$

Hence

$$
\mu \leq J\left(x_{0}, u_{0}\right) \leq \liminf _{i \rightarrow \infty} J\left(x_{l_{i}}, u_{l_{i}}\right)=\lim _{i \rightarrow \infty} J\left(x_{l_{i}}, u_{l_{i}}\right)=\mu,
$$

so

$$
J\left(x_{0}, u_{0}\right)=\mu=\inf \left\{J\left(x_{u}, u\right), \quad u \in \mathcal{U}_{M}\right\} .
$$

The proof is completed. 


\section{Illustrative example}

In this section we present the the following theoretical problems

$$
\left\{\begin{array}{l}
\left\{\begin{array}{l}
\left(-\Delta_{k}\right)^{\beta} x_{1}(t)=a \sin t\left(\sin x_{1}(t)+\sin x_{2}(t)\right)+\mathrm{e}^{t} u(t), \\
\left(-\Delta_{k}\right)^{\beta} x_{2}(t)=a \sin t\left(\sin x_{1}(t)-\sin x_{2}(t)\right)+t^{2} u(t),
\end{array} \quad t \in(0, \pi) \text { a.e. } \quad\left(\mathrm{Equ}_{\mathrm{k}}\right)\right. \\
u(t) \in[-1,1], \quad t \in(0, \pi), \\
J(x, u)=\int_{0}^{\pi}\left(\sin t+\cos t\left|\sin x_{1}(t)\right|+\sin t\left|x_{2}(t)\right|+u^{2}(t)\right) d t \rightarrow \min ,
\end{array}\right.
$$

where $k=1,2, \beta>\frac{1}{2}$ and $a>0$.

We see that $B:(0, \pi) \rightarrow \mathbb{R}^{2 \times 1}$ and

$$
B(t)=\left[\begin{array}{l}
\mathrm{e}^{t} \\
t^{2}
\end{array}\right],
$$

$$
f:(0, \pi) \times \mathbb{R}^{2} \rightarrow \mathbb{R}^{2} \text { and }
$$

$$
f\left(t, x_{1}, x_{2}\right)=\left(a \sin t\left(\sin x_{1}+\sin x_{2}\right), a \sin t\left(\sin x_{1}-\sin x_{2}\right)\right),
$$

$f_{0}:(0, \pi) \times \mathbb{R}^{2} \times[-1,1] \rightarrow \mathbb{R}$ and

$$
f_{0}\left(t, x_{1}, x_{2}, u\right)=\sin t+\cos t\left|\sin x_{1}\right|+\sin t\left|x_{2}\right|+u^{2} .
$$

It is clear that $f$ is measurable with respect to $t$, continuously differentiable on $\mathbb{R}^{2}$ and $^{4}$,

$$
\begin{aligned}
& |f(t, x)|=\left|f\left(t,\left(x_{1}, x_{2}\right)\right)\right|=\sqrt{2} a|\sin t| \sqrt{\sin ^{2} x_{1}+\sin ^{2} x_{2}} \leq 2 a+a|x|, \\
& \left|f_{x}(t, x)\right|_{2 \times 2}=\left|f_{x}\left(t,\left(x_{1}, x_{2}\right)\right)\right|_{2 \times 2}=\sqrt{2} a|\sin t| \sqrt{\cos ^{2} x_{1}+\cos ^{2} x_{2}} \leq 2 a
\end{aligned}
$$

for a.e. $t \in(0, \pi)$ and all $x \in \mathbb{R}^{2}$.

Consequently, conditions (9), (10) are satisfied with $a(t):=a, b(t):=2 a$ and $\delta(s):=$ 2. Let us note that conditions (11), (12) hold if

$$
a<\left\{\begin{array}{lll}
\frac{1}{\sqrt{2 \zeta(4 \beta)}}, & \text { if } & k=1 \\
\frac{1}{4 \beta \sqrt{2 \zeta(4 \beta)}}, & \text { if } & k=2 .
\end{array}\right.
$$

Moreover, if

$$
a \leq \begin{cases}\frac{1}{4 \zeta(2 \beta)}, & \text { if } \quad k=1 \\ \frac{1}{4^{\beta+1} \zeta(2 \beta)}, & \text { if } \quad k=2\end{cases}
$$

$\overline{{ }^{4}|\cdot| \text { denotes an Euclidean norm in }} \mathbb{R}^{2}$ and $|\cdot|_{2 \times 2}$ is a matrix norm given by $|A|_{2 \times 2}=\sqrt{\sum_{i=1}^{2} \sum_{j=2}^{2} a_{i j}^{2}}$. 
then conditions (13), (14) are satisfied.

Of course, the function $f_{0}$ satisfies assumptions $3,4,5$ of Theorem 4 . The assumption 6 also holds because

$$
f_{0}(t, x, u)=f_{0}\left(t,\left(x_{1}, x_{2}\right), u\right) \geq-1-\left|x_{1}\right|-\left|x_{2}\right| \geq-1-2|x|
$$

for a.e. $t \in(0, \pi)$ and all $x \in \mathbb{R}^{2}, u \in[-1,1]$.

Consequently, we proved the following

Theorem 5 If

$$
a<\left\{\begin{array}{ll}
\min \left\{\frac{1}{\left.\sqrt{2 \zeta(4 \beta)}, \frac{1}{4 \zeta(2 \beta)}\right\},},\right. & \text { if } k=1 \\
\min \left\{\frac{1}{4^{\beta} \sqrt{2 \zeta(4 \beta)},}, \frac{1}{4^{\beta+1} \zeta(2 \beta)}\right\}, & \text { if } \quad k=2
\end{array}= \begin{cases}\frac{1}{4 \zeta(2 \beta)}, & \text { if } k=1 \\
\frac{1}{4 \beta+1 \zeta(2 \beta)}, & \text { if } \quad k=2\end{cases}\right.
$$

then problems (18) have optimal solutions $\left(\left(x_{1}, x_{2}\right), u\right) \in D\left(\left(-\Delta_{k}\right)^{\beta}\right) \times \mathcal{U}_{[-1,1]}$, $k=1,2$.

Open Access This article is licensed under a Creative Commons Attribution 4.0 International License, which permits use, sharing, adaptation, distribution and reproduction in any medium or format, as long as you give appropriate credit to the original author(s) and the source, provide a link to the Creative Commons licence, and indicate if changes were made. The images or other third party material in this article are included in the article's Creative Commons licence, unless indicated otherwise in a credit line to the material. If material is not included in the article's Creative Commons licence and your intended use is not permitted by statutory regulation or exceeds the permitted use, you will need to obtain permission directly from the copyright holder. To view a copy of this licence, visit http://creativecommons.org/licenses/by/4.0/.

\section{A Basics of self-adjoint operators in a Hilbert space}

In this section we give the necessary notions and facts concerning a theory of unbounded self-adjoint operators in a real Hilbert space (cf. [19]). More details can be found in $[1,25]$, where all results are obtained in the case of a complex Hilbert space. Nevertheless, their proofs can be reproduced (if required, with small changes) in the case of a real Hilbert space.

So, in this section we shall assume that $H$ is a real Hilbert space with a scalar product $\langle\cdot, \cdot\rangle_{H} \cdot$

\section{A.1 Self-adjoint operator}

Let $T: D(T) \subset H \rightarrow H$ be a densely defined linear operator $(\overline{D(T)}=H)$ with the domain $D(T)$. We define

$$
D\left(T^{*}\right):=\left\{x \in H ; \quad \exists_{z \in H} \quad\langle x, T y\rangle_{H}=\langle z, y\rangle_{H}, \text { for all } y \in D(T)\right\} .
$$

For $x \in D\left(T^{*}\right)$ we denote $T^{*} x=z$ (this element is uniquely determined due to the density of $D(T)$ ). The operator $T^{*}: D\left(T^{*}\right) \subset H \rightarrow H$ is called the adjoint operator 
to $T$. If $T=T^{*}$ and $D(T)=D\left(T^{*}\right)$, then $T$ is called self-adjoint. We note that whenever $T$ is self-adjoint operator one has

$$
\langle T x, y\rangle_{H}=\langle x, T y\rangle_{H}, \text { for all } x, y \in D(T) .
$$

\section{A.2 Spectral integral and decomposition theorem}

Let $\mathcal{B}$ be a $\sigma$ - algebra of Borel subsets of $\mathbb{R}$ and $\mathcal{P}(H)$ denotes the set of all orthogonal projection operators onto closed linear subspaces of $H$. A set function $E: \mathcal{B} \rightarrow \mathcal{P}(H)$ is called a spectral measure (or a decomposition of the identity) if

1. for any $x \in H$ the set function $\mathcal{B} \ni P \rightarrow E(P) x$ is $\sigma$-additive,

2. $E(\mathbb{R})=I$ (here $I$ denotes the identity operator on $H$ ),

3. $E(P \cap Q)=E(P) \circ E(Q)$, for $P, Q \in \mathcal{B}$.

Let $W$ be the union of all open sets $V \subset \mathbb{R}$ such that $E(V)=0$. Then the complement $\mathbb{R} \backslash W$ is called a support of a spectral measure $E$ and denoted by $\operatorname{supp}(E)$.

Let assume that $u: \mathbb{R} \rightarrow \mathbb{R}$ defined a.e. $E$ is a bounded Borel measurable function. Then in the usual way one can show (via a sequence of simple functions) that for any $x \in H$ there exists the integral (with respect to the vector measure $E(\cdot) x$ )

$$
\int_{-\infty}^{+\infty} u(\lambda) E(d \lambda) x
$$

We define the integral with respect to the spectral measure $E$

$$
\int_{-\infty}^{+\infty} u(\lambda) E(d \lambda): H \rightarrow H
$$

in the following way

$$
\left(\int_{-\infty}^{+\infty} u(\lambda) E(d \lambda)\right) x=\int_{-\infty}^{+\infty} u(\lambda) E(d \lambda) x
$$

One proves that the above operator is linear, continuous and Hermitian.

Now, let $u: \mathbb{R} \rightarrow \mathbb{R}$ defined a.e. $E$ be an unbounded Borel measurable function. Let us define the sequence of functions $u_{n}$ :

$$
u_{n}(\lambda)=\left\{\begin{array}{lll}
u(\lambda), & \text { if } & |u(\lambda)| \leq n \\
0, & \text { if } & |u(\lambda)|>n
\end{array}\right.
$$


Functions $u_{n}$ are Borel measurable and bounded. Consequently, there exist integrals

$$
\int_{-\infty}^{+\infty} f_{n}(\lambda) E(d \lambda), \quad n \in \mathbb{N}
$$

Let us consider the set

$$
D=\left\{x \in H: \int_{-\infty}^{+\infty}|u(\lambda)|^{2}\|E(d \lambda) x\|_{H}^{2}<\infty\right\} .
$$

One can show that $D$ is a dense linear subspace of $H$ and for $x \in D$ there exists the limit

$$
\lim _{n \rightarrow \infty} \int_{-\infty}^{+\infty} u_{n}(\lambda) E(d \lambda) x
$$

So, we can define the operator $\int_{-\infty}^{+\infty} u(\lambda) E(d \lambda): D \subset H \rightarrow H$ in the following way:

$$
\left(\int_{-\infty}^{+\infty} u(\lambda) E(d \lambda)\right) x=\lim _{n \rightarrow \infty} \int_{-\infty}^{+\infty} u_{n}(\lambda) E(d \lambda) x .
$$

One can prove that

$$
\left\|\int_{-\infty}^{+\infty} u(\lambda) E(d \lambda) x\right\|_{H}^{2}=\int_{-\infty}^{+\infty}|u(\lambda)|^{2}\|E(d \lambda) x\|_{H}^{2}
$$

and the operator $\int_{-\infty}^{+\infty} u(\lambda) E(d \lambda)$ is self-adjoint.

If $u: \mathbb{R} \rightarrow \mathbb{R}$ is a Borel measurable function and $\omega \in \mathcal{B}$ then

$$
\int_{\omega} u(\lambda) E(d \lambda):=\int_{-\infty}^{+\infty} \chi_{\omega}(\lambda) u(\lambda) E(d \lambda),
$$

where $\chi_{\omega}$ is a characteristic function of the set $\omega$.

In order to define the spectral integral in the case of a Borel measurable function $u: P \rightarrow \mathbb{R}$, where $P \in \mathcal{B}$ contains the support $\operatorname{supp}(E)$, it is sufficient to extend $u$ on $\mathbb{R}$ to any Borel measurable function.

Now, we formulate a spectral decomposition theorem which plays a crucial role in the spectral theory of self-adjoint operators. 
Theorem 6 (Spectral decomposition theorem for self-adjoint operators) Let $T$ : $D(T) \subset H \rightarrow H$ be a self-adjoint operator such that the resolvent set $\rho(T)$ is non-empty. Then there exists a unique spectral measure $E$ with the closed support $\operatorname{supp}(E)=\sigma(T)$ such that

$$
T=\int_{-\infty}^{+\infty} \lambda E(d \lambda)=\int_{\sigma(T)} \lambda E(d \lambda) .
$$

In conclusion of this section we shall define a function of a self-adjoint operator. Let $T: D(T) \subset H \rightarrow H$ be a self-adjoint operator with $\rho(T) \neq \emptyset$. From Theorem 6 it follows that $T$ has the integral representation given by (22). For a Borel measurable function $u: \mathbb{R} \rightarrow \mathbb{R}$ defined a.e. $E$ we define the operator $u(T)$ as follows

$$
u(T)=\int_{-\infty}^{+\infty} u(\lambda) E(d \lambda)=\int_{\sigma(T)} u(\lambda) E(d \lambda)
$$

According to general properties of the spectral integrals presented above, the domain $D(u(T))$ is given by (20), the equality (21) holds and $u(T)$ is self-adjoint. Moreover, its spectrum is given by

$$
\sigma(u(T))=\overline{u(\sigma(T))}
$$

provided that $u$ is continuous on $\sigma(T)$.

\section{References}

1. Alexiewicz, A.: Functional Analysis. PWN, Warsaw (1969). (in Polish)

2. Applebaum, D.: Lévy processes - from probability to finance and quantum groups. Not. Amer. Math. Soc. 51, 1336-1347 (2004)

3. Barrios, B., Colorado, E., de Pablo, A., Sánchez, U.: On some critical problems for the fractional Laplacian operator. J. Differ. Equ. 252, 6133-6162 (2012)

4. Bates, P. W.: On some nonlocal evolution equations arising in materials science. In: Nonlinear dynamics and evolution equations, Fields Inst. Commun., vol. 48, pp. 13-52. American Mathematical Society, Providence, RI, (2006)

5. Bermudez, A., Saguez, C.: Optimal control of a Signorini problem. SIAM J. Control Optim. 25, 576-582 (1987)

6. Bogdan, K., Byczkowski, T.: Potential theory of Schrödinger operator based on fractional Laplacian. Probab. Math. Stat. 20(2), 293-335 (2000)

7. Bogdan, K., Byczkowski, T., Kulczycki, T., Ryznar, M., Song, R., Vondracek, Z.: Potential Theory of Stable Processes and its Extensions. Lecture Notes in Mathematics 1980. Springer, Berlin, Heidelberg (2009)

8. Bonforte, M., Vázquez, J. L.: A priori estimates for fractional nonlinear degenerate diffusion equations on bounded domains. The Royal Swedish Academy of Sciences, Mittag-Leffler Institute, Report No. 21, 2013/2014 arXiv:1311.6997

9. Bors, D.: Optimal control of systems governed by fractional Laplacian in the minimax framework. Int. J. Control (2019). https://doi.org/10.1080/00207179.2019.1662091 
10. Brezis, H.: Functional Analysis, Sobolev Spaces and Partial Differential Equations. Springer, New York (2011)

11. Cabré, X., Tan, J.: Positive solutions of nonlinear problems involving the square root of the Laplacian. Adv. Math. 224, 2052-2093 (2010)

12. Caffarelli, L.A., Salsa, S., Silvestre, L.: Regularity estimates for the solution and the free boundary of the obstacle problem for the fractional Laplacian. Invent. Math. 171, 425-461 (2008)

13. Caffarelli, L.A., Vasseur, A.: Drift diffusion equations with fractional diffusion and the quasigeostrophic equation. Ann. Math. 171, 1903-1930 (2010)

14. Chen, Z.-Q., Song, R.: Two-sided eigenvalue estimates for subordinate Brownian motion in bounded domains. J. Funct. Anal. 226, 90-113 (2005)

15. Cont, R., Tankov, P.: Financial Modelling with Jump Processes, Chapman \& Hall/CRC Financial Mathematics Series. Chapman \& Hall/CRC, Boca Raton, FL (2004)

16. Di Nezza, E., Palatucci, G., Valdinoci, E.: Hitchhiker's guide to the fractional Sobolev spaces. Bull. Sci. Math. 136(5), 521-573 (2012)

17. Grubb, G.: Fractional Laplacians on domains, a development of Hörmander's theory of $\mu$-transmission pseudodifferential operators. Adv. Math. 268, 478-528 (2015)

18. Idczak, D.: On a generalization of a global implicit function theorem. Adv. Nonlinear Stud. 16(1), 87-94 (2016)

19. Idczak, D.: Sensitivity of a nonlinear ordinary BVP with fractional Dirichlet-Laplace operator. arXiv:1812.11515 [math.CA] (2018)

20. Kamocki, R.: On the existence of optimal solutions to fractional optimal control problems. Appl. Math. Comput. 235, 94-104 (2014)

21. Kamocki, R.: Necessary optimality conditions for Lagrange problems involving ordinary control systems described by fractional Laplace operators. Accepted for publication in Nonlinear Analysis: Modelling and Control

22. Kisielewicz, M.: Differential Inclusions and Optimal Control. PWN, Warsaw (1991)

23. Landkof, N.: Foundations of Modern Potential Theory. Springer-Verlag, New York, (1972) (Translated from Russian by A.P. Doohovskoy)

24. Łojasiewicz, S.: An Introduction to the Theory of Real Functions. PWN, Warsaw (1973). (in Polish)

25. Mlak, W.: An Introduction to the Hilbert space. PWN, Warsaw (1970). (in Polish)

26. Olech, C.: A characterization of $L^{1}$-weak lower semicontinuity of integral functionals. Bull. Acad. Pol. Sci. 25, 135-142 (1977)

27. Schilling, R. L., Song, R., Vondracek, Z.: Bernstein Functions: Theory and Applications, 2nd edn, De Gruyter, Studies in Mathematics 37, Berlin, (2012)

28. Tan, J.: The Brezis-Nirenberg type problem involving the square root of the Laplacian. Calc. Var. 42, 21-41 (2011)

29. Vázquez, J. L.: Nonlinear diffusion with fractional Laplacian operators. Nonlinear Partial Differential Equations, vol. 7 of Abel Symposia, pp. 271-298 (2012)

30. Vázquez, J.L.: Recent progress in the theory of nonlinear diffusion with fractional Laplacian operators. Discrete Contin. Dyn. Syst. Ser. S 7, 857-885 (2014)

Publisher's Note Springer Nature remains neutral with regard to jurisdictional claims in published maps and institutional affiliations. 